\section{Sur les fonctions indépendantes III.}

\section{Par}

\section{J. Marcinkiewicz (Wilno).}

1. Cette partie contient la suite des recherches publiées sous le même titre dans deụx parties précédentes ${ }^{1}$ ). Elle concerne le problème suivant:

Etant donné, pour tout $n$, un système de fonctions indépendantes

$$
x_{n, 1}, x_{n, 2}, x_{n, 3}, \ldots
$$

telles que les distribuantes des sommes

$$
\sum_{\nu} x_{n, v}
$$

convergent vers une distribuante $\nabla$, quel est le caractère de cette convergence lorsque la fonstion caractéristique de la distribuante $\nabla$ est analytique?

2. Soit $V(x)$ une distribuante et

$$
\varphi(t)=\int_{-\infty}^{+\infty} e^{i t x} d V(x)
$$

sa fonction caractéristique. La question s'impose sous quelles conditions concernant $V(x)$ la fonction $\varphi(t)$ est analytique dans un entourage du point 0 . Un intérêt paraît présenter aussi la question dans quels cas $\varphi(t)$ est fonction frontière d'une fonction analytique. Je vais démontrer à ce sujet le

1) Fund. Math. 30 (1938), p. 202-214 et 349-364. Dans la I partie, j'ai commis une erreur dans l'énoncé du théorème 3, p. 209. Je la corrige à la fin de la partie présente, p. 101, Errata.
Théorème 1. Pour que la fonction $p(x)$ où $|x|<\Delta$ soit fonction frontière d'une fonction analytique régulière dans le rectangle

$$
0 \leqslant I(z) \leqslant R, \quad|R(z)| \leqslant \Delta,
$$

il faut et il suffit que l'intégrale

$$
\int_{-\infty}^{0} e^{-r x} d V(x)
$$

existe pour tout $r<R$.

On voit d'abord que cette condition est suffisante. En effet, la fonction

$$
\varphi_{1}(z)=\int_{-\infty}^{0} e^{i z t} d V(t)
$$

est régulière pour $I(z)<R$. D'autre part, la fonction

$$
\varphi_{2}(z)=\int_{0}^{\infty} e^{i z t} d V(t)
$$

est analytique pour $I(z)>0$, ce qui montre que la fonction

$$
\varphi(z)=\varphi_{1}(z)+\varphi_{2}(z)
$$

est analytique dans la bande $0<I(z)<R$ et que ses valeurs limites pour $z \rightarrow x, I(z)>0$ coïncident avec $\varphi(x)$.

La démonstration que la condition est nécessaire sera basée sur 5 lemmes:

\section{Lemme 1. Soit}

$$
\varphi(x)=\int_{-\infty}^{+\infty} e^{i x t} d V(x)
$$

Pour que $\varphi^{(k)}(0)$ avec k pair existe, il faut et il suffit que l'on ait

$$
\int_{-\infty}^{+\infty} x^{k} d V(x)<\infty .
$$

La condition (2.4) étant vérifiée, on a

$$
\varphi^{(k)}(0)=i_{-\infty}^{k} \int_{-\infty}^{+\infty} x^{k} d V(x) .
$$

Ce lemme est connu ${ }^{2}$ ).

2) P. Lévy [6], p. 174-175. Pour $k$ impair, la proposition n'est pas vraie, comme on le verifie facilement en posant $k=1$ et $V(x)=$ const. $\int_{-\infty}^{x} \frac{d u}{\left(1+u^{2}\right) \log \left(2+u^{2}\right)}$. 
Lemme 2. Pour que la fonction $\varphi(x)$, définie par la formule (2.3), soit régulière dans le cercle $|z|<R$, il faut et il suffit que l'on ait pour tout $r<R$

$$
\int_{-\infty}^{+\infty} e^{r|x|} d V(x)<\infty
$$

Ce lemme est aussi connu ${ }^{3}$ ). Nous en donnons ici la démonstration pour la commodité du lecteur. Posons:

Il ne s'agit que de démontrer la nécessité de l'inégalité (2.6).

$$
M_{n}^{*}=\int_{-\infty}^{+\infty}|x|^{n} d V(x), \quad M_{n}=\int_{-\infty}^{+\infty} x^{n} d V(x)
$$

En tenant compte de (2.5) et du fait que la fonction $\varphi(x)$ est analytique pour $|z|<R$, on a

$$
\sum \frac{1}{n !}\left|M_{n}\right| r^{n}<\infty \quad 0<r<R .
$$

Or, comme

$$
M_{2 n-1}^{*} \leqslant M_{2 n}^{*}{ }^{\frac{2 n-1}{2 n}}, \quad M_{2 n}=M_{2 n}^{*}
$$

l'inégalité (2.7) donne aussi

$$
\sum \frac{1}{n !} M_{n}^{*} r^{n}<\infty \quad 0<r<R,
$$

ce qui est bien la condition (2.6).

Lemme 3. Lorsque la fonction $\varphi(x)$ est régulière pour $|z|<R$, elle est regulière dans la bande $|I(z)|<R$.

Cela résulte immediatement de l'inégalité (2.6).

Lemme 4. Si la fonction $\varphi(z)$ est analytique dans le rectangle

$$
|R(z)|<\Delta, \quad|I(z)|<R
$$

elle l'est dans la bande $|I(z)|<R$.

Désignons par $r$ le rayon du plus grand cercle de centre 0 dans lequel la fonction $\varphi(z)$ est encore régulière. D'après le lemme 3 , il suffit de montrer que $r=R$. Supposons par contre que $r<R$. La fonction $\varphi(z)$ étant, d'après le lemme 3 , régulière pour $|I(z)|<r$ et, par hypothèse, dans le rectangle (2.8), elle est aussi régulière dans le cercle de rayon $\varrho=\min \left(R, \sqrt{\left.r^{2}+\Delta^{2}\right)}\right.$, ce qui entraîne $r=R$.

3) P. Lévy [7], p. 41; ef. aussi G. Pólya [12].

Lemme 5. Soit $D$ le rectangle (2.1) et $D^{*}$ le rectangle symétrique $\grave{a} D$ par rapport à l'axe des $x$. Lorsque une fonction $\psi(z)$ est régulière dans les rectangles $D$ et $D^{*}$ et continue dans l'intervalle $\langle-\Delta,+\Delta\rangle$, elle est régulière dans tout rectangle (2.8).

Il est difficile de dire si ce lemme a été formulé explicitement dans la littérature. En tout cas, il est étroitement lié au principe de symétrie de Schwarz. Pour l'établir, considérons la fonction

$$
\Psi(z)=\int_{R} \frac{\psi(\xi)}{z-\xi} d \xi
$$

où $R$ désigne le contour du rectangle (2.8). Cette fonction est évidemment analytiqque. D'autre part, il est facile d'établir l'égalité

$$
\Psi(z)=\int_{R_{1}} \frac{\psi(\xi)}{z-\xi} d \xi+\int_{R_{z}} \frac{\psi(\xi)}{z-\xi} d \xi
$$

où $R_{1}$ et $R_{2}$ désignent respectivement les contours des rectangleś $D$ et $D^{*}$. La dernière égalité montre que $\Psi(z)=\psi(z)$ lorsque $z \epsilon D$, ce qui achève la démonstration.

Pour démontrer à présent la nécessité de l'existence de l'intégrale (2.2), posons

$$
\begin{aligned}
\varphi(x) & =\varphi_{1}(x)+\varphi_{2}(x) \\
\text { où } \varphi_{1}(x)=\int_{0}^{\infty} e^{i x t} d V(t) \text { et } \varphi_{2}(x) & =\int_{-\infty}^{0} e^{i x t} d V(t) .
\end{aligned}
$$

La fonction $\varphi(x)$ est régulière dans le rectangle (2.1) et la fonction $\varphi_{1}(x)$ l'est dans le demi-plan $I(z)>0$. Il en résulte que la fonction $\varphi_{2}(x)$ est régulière dans le rectangle (2.1). D'autre part, elle est d'une façon évidente régulière dans le demi-plan $I(z)<0$, ce qui montre d'après le lemme 5 qu'elle l'est aussi dans le rectangle (2.8). Or, $\varphi_{2}(x)$ ne diffère d'une fonction caractéristique que par un facteur constant; d'après le lemme 2, il en résulte facilement l'existence de l'intégrale (2.2) pour $r<R$, c. q.f. d.

3. Comme conséquence immédiate $d u$ th. 1 on a le

Theorème 2. Soient: $\nabla_{n}(x)$ une suite de distribuantes et $\varphi_{n}(x)$ la suite de leurs fonctions caractéristiques. Pour que $\nabla_{n}$ converge vers une distribuante $V$ dont la fonction caractéristique $\varphi(x)$ est fonction frontière d'une fonotion régulière dans le rectangle (2.1), il faut et il suffit que la suite $\varphi_{n}(x)$ converge vers $\varphi(x)$ dans un intervalle $\langle-\Delta,+\Delta\rangle$. 
Remarquons d'abord que ce théorème cesse d'être vrai si la fonction $\varphi(x)$ est arbitraire $\left.{ }^{4}\right)$. En effet, considérons deux fonctions suivantes $\left.{ }^{5}\right)$ :

$$
\psi(x)=\left\{\begin{array}{cc}
(1-|x|)^{2} & \text { si }|x| \leqslant 1 \\
0 & \text { si }|x|>1,
\end{array} \quad \psi_{1}(x)=\left\{\begin{array}{c}
(1-|x|)^{2} \text { si }|x|<1 \\
\text { et de période } 1 .
\end{array}\right.\right.
$$

On vérifie facilement que ce sont des fonctions caractéristiques. Elles coincident dans l'intervalle $\langle-1,+1\rangle$ et leurs distribuantes sont tout à fait différentes.

Il ne s'agit, enfin, que de démonter que la condition énoncée est suffisante.

Or, la suite de fonctions caractéristiques $\psi(x) \varphi_{n}(x)$ converge partout vers la fonction caractéristique $\psi(x) \varphi(x)$, ce qui montre bien que leurs distribuantes convergent vers $\int_{-\infty}^{+\infty} G(x-u) d V(u)$, où $G(x)$ désigne la distribuante dont la fonction caractéristique est $\psi(x)$. On en tire facilement:

$$
V_{n}(x)<\varepsilon \text { pour } x<-R, \quad 1-V_{n}(x)<\varepsilon \text { pour } x>R,
$$

dès que $R$ et $n$ sont suffisamment grands. La relation (3.2) montre que la suite $V_{n}(x)$ forme une famille normale. En supposant que la suite $\nabla_{n}(x)$ ne converge pas vers $V$, on pourrait en extraire une suite $V_{n_{i}}(x)$, convergente vers une distribuante

$$
\nabla^{*} \neq V \text {. }
$$

Soit $\varphi^{*}$ sa fonction caractéristique. On a $\varphi^{*}(x)=\varphi(x)$ pour $|x|<\Delta$. La fonction $\varphi(x)$ étant fonction frontière d'une fonction analytique, $\varphi^{*}(x)$ l'est aussi. D'après le lemme $4, \varphi^{*}(x)$ serait donc une fonction frontière dans toute la bande $0<g(z)<R$ et, par conséquent, elle coinciderait avec $\varphi(x)$ pour tout $x$. On aurait done $V^{*}=V$, contrairement à (3.3).

Soulignons quelques cas importants de ce théorèmẹ général.

Théorème 3. Soit $\nabla$ une distribuante, $\nabla(0-0)=0$ et $\varphi(x)$ la fonction caractéristique de $V$. Pour que la suite de distribuantes $V_{n}$ converge vers la distribuante $V$, il faut et il suffit que la suite des fonctions caractéristiques de $V_{n}$ converge vers $\varphi(x)$ dans un intervalle $\langle-\Delta,+\Delta\rangle$.

\footnotetext{
4) H. Cramér [3], p. 29 et 121, P. Lévy [7], p. 49.
}

5) données par A. Khintchine; voir P. Lévy [7], p. 190.
Théorème $4^{6}$ ). Pour que la suite des distribuantes $\nabla_{n}(x)$ converge vers la distribuante de Gauss, il faut et il suffit que la suite de leurs fonctions caractéristiques converge vers $e^{-x^{2} / 2}$ dans un intervalle $\langle-\Delta,+\Delta\rangle$.

4. Soit $x(t)$ une fonction définie dans l'intervalle $\langle 0,1\rangle$. On appelle sa médiane ${ }^{7}$ ) le nombre $\lambda$ satisfaisant aux conditions:

$$
|\underset{t}{\mathrm{E}}(x(t) \leqslant \lambda)| \geqslant \frac{1}{2}, \quad \underset{t}{\mathrm{E}}(x(t) \geqslant \lambda) \mid \geqslant \frac{1}{2} .
$$

Cette notion nous paraît très commode. En général la médiane n'est pas définie d'une manière univoque. Pour éviter cet inconvénient, on peut prendre comme médiane la moyenne arithmétique du plus petit et du plus grand des nombres satisfaisant aux inégalités (4.1), mais c'est sans importance pour les calculs que nous allons faire.

5. Théorème 5. Soit $V(x)$ une distribuante dont la fonction caractéristique $\varphi(x)$ est régulière dans la bande $-R<I(z)<+R$. Pour que la distribuante de la somme

$$
\sum_{\nu} x_{n, v}
$$

ò̀ les fonctions $x_{n, v}$ sont indépendantes, converge vers $\nabla(x)$, il faut et il suffit qu'on puisse choisir des constantes $K_{1}, K_{2}, \ldots$ de facon que la suite des fonctions caractéristiques $\left\{\varphi_{n}^{*}(x)\right\}$ des sommes

ò̀

$$
x_{n, v}^{*}=\left\{\begin{array}{lll}
x_{n, v} & \text { pour } & \left|x_{n, v}-\lambda_{n, \nu}\right| \leqslant K_{n} \\
\lambda_{n, v} & \text { pour } & \left|x_{n, \nu}-\lambda_{n, v}\right| \geqslant K_{n},
\end{array}, \sum_{v}\left|\mathrm{E}\left(\left|x_{n, \nu}-\lambda_{n, \nu}\right|>K_{n}\right)\right| \rightarrow 0\right.
$$

et $\lambda_{n, v}$ désigne la médiane de la fonction $x_{n, v}$, converge uniformément vers $\varphi(z)$ dans tout rectangle

$$
|I(z)| \leqslant r, \quad r<R, \quad|R(z)| \leqslant \Delta .
$$

Il ne s'agit que de démontrer la nécessité des conditions énoncées. Nous allons d'abord démontrer trois lemmes.

6) Cf. P. Lévy [7], p. 41.

7) A. Cournot [2], p. 120. 


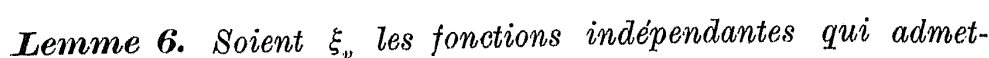
tent les valeurs \pm 1 dans les ensembles de mesure $\delta_{\nu}$ et sont égales $\dot{a} 0$ dans les autres points de l'intervalle $\langle 0,1\rangle$. Posons

et soit

$$
M_{n}^{*}=\int_{0}^{1}\left|\sum_{v} \xi_{v}\right|^{n} d t
$$

\section{Alors}

$$
\sum \delta_{\nu} \leqslant H
$$

$$
M_{n}^{*} \leqslant n ![M / \log n]^{n}, \quad M=M(H) .
$$

La fonction caractéristique de $\xi_{p}$ est évidemment

$$
1-2 \delta_{\nu}+\delta_{\nu}\left\{e^{i z}+e^{-i z}\right\}
$$

ce qui donne pour la fonction caractéristique de la somme $\sum_{\nu} \xi_{n}$ l'expression $\Phi(z)=\prod_{\nu}\left\{1-2 \delta_{\nu}+\delta_{\nu}\left(e^{i z}+e^{-i z}\right)\right\}$. Il en résulte que

$$
|\Phi(z)| \leqslant \prod_{\nu}\left(1+2 \delta_{\nu}+2 \delta_{\nu} e^{|z|}\right) \leqslant \prod_{\nu}\left(1+4 \delta_{\nu} e^{|z|}\right) \leqslant e^{4 H e^{|z|}} .
$$

Quel que soit $R$, on trouve d'après le théorème de Cauchy $\left|\Phi^{(n)}(0)\right| \leqslant n ! e^{4 H e^{R}} \cdot R^{-n}$. En y posant $R=\log n$, on obtient

$$
\left|\dot{\Phi}^{(n)}(0)\right| \leqslant n ! e^{4 H n} \lg n^{-n} \text {. }
$$

D'autre part, $\left|\Phi^{2 s}(0)\right|=M_{2 s}^{*}$, d'où $M_{2 s}^{*} \leqslant(2 s) ! e^{8 H s} \lg s^{-2 s}$ et on en déduit facilement l'inégalité (5.6).

Lemme \%. Soient $x_{\nu}$ indépendantes et symétriques. Supposons de plus qu'on a:

On a alors

$$
\begin{gathered}
\left|x_{\nu}\right| \leqslant K \\
\sum_{\nu} \int_{0}^{1} x_{\nu}^{2} d t \leqslant H .
\end{gathered}
$$

$$
\int_{0}^{1}\left|\sum_{\nu} x_{\nu}\right|^{n} d t \leqslant(1+K)^{n} n ![M / \lg n]^{n}
$$

où $M$ ne dépend qüe de $H^{8}$ ).

8) Ce lemme peut être démontré par voie directe, en partant de l'égalité

$$
\varphi_{\nu}(z)=\sum_{n} \frac{i^{n} z^{n}}{n !} \int_{0}^{1} x_{\nu}^{n} d t
$$

mais la méthode utilisée ici paraît présenter un certain intérêt par elle-même.
Il suffit de l'établir pour $n=2 s$. On a

$$
\int_{0}^{1}\left(\sum_{v} x_{\nu}\right)^{2 s} d t=\sum_{\nu} \int_{0}^{1} x_{\mu_{1}} \cdot \ldots \cdot x_{\mu_{2 s}} d t=\sum_{\nu} B \int_{0}^{1} x_{\nu_{1}}^{2 \alpha_{1}} d t \ldots \int_{0}^{1} x_{\nu_{2 s}}^{2 \alpha_{2 s}}
$$

où $\quad \nu_{1} \neq \nu_{2} \neq \ldots \neq \nu_{2 s}, \quad \sum_{i} 2 \alpha_{i}=2 s$ et $B$ désignent les coefficients de Newton. Supposons que $K \leqslant 1$ et posons $2 \delta_{\nu}=\int_{0}^{1} x_{v}^{2} d t$. Soit $\xi_{v}$ une fonction égale ̀̀ \pm 1 dans des ensembles de mesure $\delta_{y}$ et à 0 dans les autres points de l'intervalle $\langle 0,1\rangle$. Il est évident que

$$
\int_{0}^{1} x_{\nu}^{2 s} d t \leqslant \int_{0}^{1} \xi_{\nu}^{2 s} \quad \nu=1,2, \ldots ; s=0,1, \ldots
$$

Il en résulte que

$\int_{0}^{1}\left|\sum_{\nu} x_{\nu}\right|^{2 s} d t \leqslant \sum_{\nu} B \int_{0}^{1} \xi_{\nu_{1}}^{2 \alpha_{1}} d t \ldots \int_{0}^{1} \xi_{\nu_{2 s}}^{2 \alpha_{2 s}} d t, \quad$ c.̀̀d d. $\int_{0}^{1}\left|\sum_{\nu} x_{\nu}\right|^{2 s} d t \leqslant \int_{0}^{1}\left|\sum_{\nu} \xi_{\nu}\right|^{2 s} d t$, ce qui donne en vertu de (5.6)

$$
\int_{0}^{1}\left|\sum x_{\nu}\right|^{2 s} d t \leqslant(2 s) ![M / \lg s]^{2 s}
$$

On en déduit facilement (5.9) lorsque $K \leqslant 1$. Lorsque $K>1$, il suffit d'appliquer ce résultat aux fonctions $x_{v} / K$.

En s'appuyant sur ce lemme, nous allons démontrer le

Lemme 8. Le théorème 5 est vrai, lorsque les fonctions $x_{n, v}$ sont symétriques.

D'après le th. 3 de la I partie de ce travail ${ }^{9}$ ), on peut supposer que

$$
\int_{0}^{1}\left|\sum_{\nu} x_{n, \nu}\right|^{2 s} d t \rightarrow M_{2 s} \quad s=1,2, \ldots
$$

où $M_{2 s}$ désigne le moment d'ordre $2 s$ de la distribuante limite $\nabla(x)$. En particulier,

$$
\sum_{\nu} \int_{0}^{1} x_{n, \nu}^{2} d t \leqslant H
$$

๑) J. Matoinkiewicz [8]; voir aussi Errata à la fin de la partie présente (p. 101). 
Il vient de là facilement

$$
\sum_{t}\left|\mathrm{E}\left(\left|x_{n, v}\right|>K\right)\right| \leqslant H K^{-2} .
$$

Soit $K_{n}$ une suite quelconque satisfaisant à l'unique condition $K_{n} \rightarrow \infty$. Soit

$$
x_{n, \nu}^{*}=\left\{\begin{array}{ccc}
x_{n, \nu} & \text { pour } & \left|x_{n, \nu}\right| \leqslant K_{n} \\
0 & \text { pour } & \left|x_{n, \nu}\right|>K_{n} .
\end{array}\right.
$$

En tenant compte de (5.11), on conclut facilement que la distribuante de la somme $\sum_{\nu} x_{n, \nu}^{*}$ converge vers $V$; de plus, on a

$$
\int_{0}^{1}\left|\sum_{\nu} x_{n, \nu}^{*}\right|^{2 s} d t \leqslant \int_{0}^{1}\left|\sum_{\nu} x_{n, \nu}\right|^{2 s} d t
$$

En effet:

$$
\begin{aligned}
& \int_{0}^{1}\left|\sum_{\nu} x_{n, \nu}^{*}\right|^{2 s} d t=\sum_{\nu} B \int_{0}^{1} x_{n, \nu_{1}}^{* 2 \alpha_{1}} d t \ldots \int_{0}^{1} x_{n, \nu_{2 s}}^{* 2 \alpha_{2 s}} d t \\
& \int_{0}^{1}\left|\sum_{\nu} x_{n, \nu}\right|^{2 s} d t=\sum_{\nu} B \int_{0}^{1} x_{n, \nu_{1}}^{2 \alpha_{1}} d t \ldots \int_{0}^{1} x_{n, \nu_{2 s}}^{2 \alpha_{2 s}}
\end{aligned}
$$

et, pour en tirer (5.12), il suffit comparer les termes correspondants des formules (5.13) et (5.14).

Désignons par $\left\{n_{s}\right\}$ une suite croissante telle que

$$
\int_{0}^{1}\left|\sum_{\nu} x_{n, \nu}\right|^{2 r} d t \leqslant 2 M_{2 r} \quad n \geqslant n_{s} ; 1 \leqslant r \leqslant s !
$$

et posons

$$
K_{r}=\sqrt{s} \quad \text { pour } \quad n_{s} \leqslant r<n_{s+1} .
$$

On a pour $n_{s} \leqslant n \leqslant n_{s+1}$ :

$$
\begin{array}{cc}
\int_{0}^{1}\left|\sum_{v} x_{n, \nu}^{*}\right|^{2 \lambda} d t \leqslant 2 M_{2 \lambda} & 1 \leqslant \lambda \leqslant s ! \\
\int_{0}^{1}\left|\sum_{\nu} x_{n, \nu}^{*}\right|^{2 \lambda} d t \leqslant(2 \lambda) !\left(1+K_{r}\right)^{2 \lambda}[M / \log \lambda]^{2 \lambda} & \lambda=2,3, \ldots
\end{array}
$$

La dernière formule donne pour $\lambda>s$ !

(5.17) $\int_{0}^{1}\left|\sum_{\nu} x_{n, v}^{*}\right|^{22} d t \leqslant(2 \lambda) !\left(1+K_{r}\right)^{2 \lambda}[M / s]^{22} \leqslant(2 \lambda) ![2 M / / \bar{s}]^{2 \lambda}$.
Il en vient pour $a$ réels, où $|a|<R$ et $n_{s} \leqslant n \leqslant n_{s+1}$ :

$$
\begin{gathered}
\int_{0}^{1} \exp a \sum_{\nu} x_{n, \nu}^{*} d t=\sum_{0}^{\infty} \frac{1}{(2 s) !} a^{2 s} \int_{0}^{1}\left(\sum_{\nu} x_{n, \nu}\right)^{2 s} d t=\sum_{1}^{n_{s}}+\sum_{n_{s}+1}^{\infty} \leqslant \\
\leqslant 2 \sum_{1}^{n_{s}} \frac{1}{(2 i) !} a^{2 i} M_{2 i}+\sum_{n_{s}+1}^{\infty}\left[\frac{2 M}{V_{s}}\right]^{2 i} a^{2 i}=A+B .
\end{gathered}
$$

Or, $a$ étant fixé, on a pour $n$ suffisament grands $|(2 M / V s) a| \leqslant \frac{1}{2}$, ce qui donne $B \leqslant 2$. On en tire pour $n>n_{a}$

$$
\int_{0}^{1} \exp a \sum_{\nu} x_{n, \nu}^{*} d t \leqslant 2+2 \int_{0}^{\infty} \exp a x d V(x) .
$$

On voit done que les fonctions $\varphi_{n}^{*}(z)$ sont uniformément bornées dans chaque bande $|I(z)|<r$ où $r<R$; comme elles convergent vers $\varphi(x)$ sur l'axe réelle, il en résulte d'après un théorème elassique qu'elles convergent uniformément dans tout rectangle (5.4).

6. Oeci établi, la démonstration du th. 5 s'achève comme il suit. Soient $x_{n, v}$ et $x_{n, v}^{\prime}$ indépendantes $(n, v=1,2, \ldots)$ et $x_{n, v}$ équimesurable avec $-x_{n, \nu}$. D'après le th. 3 de la I partie de cet ouvrage ${ }^{9}$ ), nous pouvons trouver des constantes $K_{1}, K_{2}, \ldots$ de façon qu'en posant

$$
\omega_{n, \nu}=\left\{\begin{array}{ccc}
x_{n, v}+x_{n, \nu}^{\prime} & \text { pour } & \left|x_{n, \nu}+x_{n, \nu}^{\prime}\right| \leqslant K_{n} \\
0 & \text { pour } & \left|x_{n, \nu}+x_{n, \nu}^{\prime}\right|>K_{n}
\end{array}\right.
$$

on ait:

$$
\int_{0}^{1}\left(\sum_{\nu} \omega_{n, v}\right)^{2} d t \leqslant H, \quad \sum_{v}\left|\mathrm{E}\left(\left|x_{n, v}+x_{n, v}^{\prime}\right|>K_{n}\right)\right| \rightarrow 0
$$

ce qui donne

$$
\sum_{\nu} \int_{0}^{1} \omega_{n, v}^{2} d t \leqslant H
$$

Il vient pour tout $K$

$$
\sum_{\nu}\left|\underset{t}{\mathrm{E}}\left(\left|x_{n, \nu}+x_{n, v}^{\prime}\right|>K\right)\right| \leqslant H K^{-2}+o(1) .
$$

En désignant par $\lambda_{n, v}$ la médiane de la fonction $x_{n, v}$, on en tire 
Cette inégalité montre que, quelle que soit la suite $\Delta_{n} \rightarrow \infty$, la distribuante de la somme $\sum_{y} \xi_{n, y}$ où

$$
\xi_{n, v}=\left\{\begin{array}{lll}
x_{n, v} & \text { si } & \left|x_{n, v}-\lambda_{n, v}\right| \leqslant \Delta_{n} \\
\lambda_{n, v} & \text { si } & \left|x_{n, v}-\lambda_{n, v}\right|>\Delta_{n}
\end{array}\right.
$$

tend vers $V$. D'autre part, d'après le lemme 8 , on peut choisir une suite $\left\{K_{n}\right\}$ de manière qu'en posant

on ait

$$
\eta_{n, \nu}=\left\{\begin{array}{lll}
x_{n, \nu}+x_{n, v}^{\prime} & \text { si } & \left|x_{n, \nu}+x_{n, v}^{\prime}\right| \leqslant K \\
0 & \text { si } & \left|x_{n, \nu}+x_{n, v}^{\prime}\right|>K
\end{array}\right.
$$

$$
\int_{0}^{1} \exp a \sum_{v} \eta_{n_{2},} d t \leqslant M(a)
$$

$$
\begin{gathered}
\int_{0}^{1} \exp a \sum_{\nu} \eta_{n, v} d t=\sum_{v} \frac{1}{(2 s) !} \int_{0}^{1}\left(\sum_{\nu} \eta_{n, v}\right)^{2 s} d t= \\
=\sum_{s} \frac{1}{(2 s) !} \sum_{\nu} B \int_{0}^{1} \eta_{n, \nu_{1}}^{2 \alpha_{1}} d t \ldots \int_{0}^{1} \eta_{n, \nu_{2 s}}^{2 \alpha_{2 s}} d t .
\end{gathered}
$$

En posant $\Delta_{n}=K_{n} / 2$. et en définissant $\xi_{n, v}^{\prime}$ d'une façon analogue à $\xi_{n, v}$, on a pour $a>0$ :

$\int_{0}^{1}\left(\xi_{n, \nu}+\xi_{n, \nu}^{\prime}\right)^{2 \alpha} d t-\int_{\mid \xi_{n, \nu}^{\prime}-\lambda_{n, \nu}>\Delta_{n}}\left(\xi_{n, \nu}-\lambda_{n, \nu}\right)^{2 \alpha}-\int_{\left|\xi_{n, \nu}^{\prime}+\lambda_{n, \nu}\right|>\Delta_{n}}\left(\xi_{n, \nu}^{\prime}+\lambda_{n, \nu}\right)^{2 \alpha} \leqslant \int_{0}^{1} \eta_{n, \nu}^{2 \alpha} d t$.

Or, comme

$$
\begin{gathered}
\int_{\left|\xi_{n, \nu}^{\prime}+\lambda_{n, \nu}\right|}\left|\xi_{n, \nu}-\lambda_{n, \nu} \nu^{2 \alpha} d t=\right| \underset{t}{\mathrm{E}}\left(\left|\xi_{n, \nu}^{\prime}+\lambda_{n, \nu}\right|>\Delta_{n}\right)\left|\cdot \int_{0}^{1}\right| \xi_{n, \nu}-\left.\lambda_{n, \nu}\right|^{2 \alpha} d t \\
\int_{0}^{1}\left(\xi_{n, \nu}+\xi_{n, \nu}^{\prime}\right)^{2 \alpha} \geqslant \frac{1}{2} \int_{0}^{1}\left(\xi_{n, \nu}-\lambda_{n, \nu}\right)^{2 \alpha} d t
\end{gathered}
$$

on a aussi

$$
\int_{0}^{1}\left(\xi_{n, \nu}+\xi_{n, \nu}^{\prime}\right)^{2 \alpha} d t \leqslant(1+o(1)) \int_{0}^{1} \eta_{n, \nu}^{2 \alpha} d t
$$

ce qui donne d'après $(6.4)$

$$
\int_{0}^{1} \exp a \sum\left(\xi_{n, \nu}+\xi_{n, \nu}^{\prime}\right) d t=O(1) \quad|a|<R
$$

La distribuante de la somme

$$
\sum_{v} \xi_{n, v}
$$

tendant vers $V$, on peut trouver deux nombres $M$ et $\varepsilon$ tels que

$$
\left|\mathrm{E}\left[\left|\sum_{\nu} \xi_{n, \nu}^{\prime}\right| \leqslant M\right]\right|>\varepsilon
$$

La formule (6.5) donne alors

$$
\int_{0}^{1} \exp a \sum_{v} \xi_{n, v} d t=O(1)
$$

On voit done que la suite des fonctions caractéristiques des sommes (6.6) est uniformément bornée dans chaque bande $|I(z)|<r$ où $r<R$ et, étant convergente pour $z$ réel, converge nécessairement dans tout le rectangle $|I(z)|<r<R,|R(z)|<\Delta$.

7. Soient $V(x)$ une distribuante et $\varphi(x)$ sa fonction caractéristique. On dit que $\nabla(x)$ est infiniment divisible si, pour tout $n$, la fonction $\varphi^{1 / n}(x)$ est une fonction caractéristique.

La théorie des distribuantes infiniment divisibles constitue une branche importante de la théorie des probabilités.

Il nous semble assez naturel de poser le problème suivant:

Etant données, pour tout n, les fonctions indépendantes (1.1), quelles sont les conditions nécessaires et suffisantes pour que les distribuantes des sommes (1.2) convergent vers une loi infiniment divisible $\nabla(x)$ ? $\left.{ }^{10}\right)$.

Nous allons résoudre ce problème avec une précission suffisante dans deux hypothèses supplémentaires. Nous supposerons d'abord que la fonction caractéristique $\varphi(z)$ est régulière dans une bande $|I(z)|<R$ et, de plus, que

$$
\max _{v}\left|\mathrm{E}\left(\left|x_{n, v}\right|>\varepsilon\right)\right| \rightarrow 0 \text {. }
$$

Dans ces hypothèses nous allons établir ce

Théorème 6. Pour que les distribuantes $V_{n}$ des sommes $\sum_{\nu} x_{n, \nu}$, où $x_{n, v}$ vérifient les conditions (7.1), convergent vers la distribuante $\nabla$ d'une fonction caractéristique régulière dans un entourage du point 0 , il faut et il suffit que l'on puisse ohoisir de telles constantes $K_{1}, K_{2}, \ldots$ qu'en posant

10) Pour les rapports de ce problème avec les distribuantes infiniment divisibles, voir A. Khintchine [5].

Fundamenta Mathematicae. T. XXXI. 
on ait:

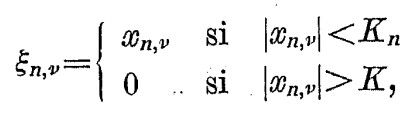

$$
\sum_{\nu}\left|\mathrm{E}\left(\left|x_{n, v}\right|>K_{n}\right)\right| \rightarrow 0,
$$

(7.3) $\quad \sum_{\nu} \int_{0}^{1} \xi_{n, \nu} d t \rightarrow \int_{-\infty}^{+\infty} x d V(x), \quad \sum_{\nu} \int_{0}^{1}\left(\xi_{n, \nu}-m_{n, \nu}\right)^{k} d t \rightarrow \sigma_{k} \quad k=2,3, \ldots$, où $m_{n, v}$ désigne la moyenne de la fonetion $x_{n, v}$ et les nombres $\sigma_{k}$ sont définis par les relations:

$$
\begin{gathered}
\operatorname{tg} e^{m z} \varphi(-i z)=\sum_{k} \frac{\sigma_{k}}{k !} z_{k}, \\
e^{m z} \varphi(i z)=\int_{-\infty}^{+\infty} e^{-z t} d V(t+m), \quad m=\int_{-\infty}^{+\infty} x d V(x) .
\end{gathered}
$$

Comme $\varphi(0)=1$, la série (7.4) admet un rayon de convergence positif. Posons $y=\lg \varphi(z)$. On a alors $y^{\prime} \varphi=\varphi^{\prime}$ et il vient

$$
\sum_{0}^{n}\left(\begin{array}{c}
n \\
i
\end{array}\right) y^{(i+1)}(0) \varphi^{(n-t)}(0)=\varphi^{(n+1)}(0) .
$$

Or, comme $\varphi^{(s)}(0)=i^{s} M_{s}$, il vient d'après (7.5)

$$
\sum_{0}^{n}\left(\begin{array}{l}
n \\
j
\end{array}\right) y^{(j+1)}(0) i^{(n-1)} M_{n-j}=i^{n+1} M_{n+1}
$$

La démonstration du th. 6 sera basée sur le

Lemme $\left.{ }^{11}\right)$. Soient $x_{n, v}$ des fonotions indépendantes. Posons:

$$
\begin{gathered}
\sigma_{k}=\sum_{\nu} \int_{0}^{1} x_{\nu}^{k} d t \\
\sigma_{k}^{*}=\sum_{\nu}\left|\int_{0}^{1} x_{\nu}^{k} d t\right|, \\
s=\sum_{\nu} x_{\nu}, \quad \delta_{k}=\max _{1 \leqslant i \leqslant k}\left|\int_{0}^{1} x_{\nu}^{i} d t\right| . \\
\int_{0}^{1} S^{k} d t=F_{k}\left(\sigma_{1}, \ldots, \sigma_{k}\right)+\Theta H_{k}\left(\delta_{1}, \ldots, \delta_{k}\right),
\end{gathered}
$$

Alors

$F_{k}$ étant définis par les formules:

$$
\begin{gathered}
\sigma_{1}=F_{1}\left(\sigma_{1}\right), \sigma_{2}+\sigma_{1}^{2}=F\left(\sigma_{1}, \sigma_{2}\right), \\
\text { (7.11) } \sigma_{k+1}+\sum_{1}^{k}\left(\begin{array}{l}
k \\
i
\end{array}\right) \sigma_{k-i+1} F_{i}\left(\sigma_{1}, \ldots, \sigma_{i}\right)=F_{k+1}\left(\sigma_{1}, \ldots, \sigma_{k+1}\right) \\
\text { ò̀ }|\Theta| \leqslant 1 \text { et } H_{k} \rightarrow 0 \text { lorsque } \max _{1 \leqslant k} \sigma_{n}^{*} \leqslant M \text { et } \max _{0 \leqslant k} \delta_{i} \rightarrow 0 .
\end{gathered}
$$

\footnotetext{
11) Cf. J. Marcinkiewicz [9], p. 355-356.
}

Pour $k=1$, ce lemme est évident. Supposons-le vrai pour $k=r$; nous allons en déduire qu'il est vrai aussi pour $k=r+1$. On a

$$
\int_{0}^{1} S^{r+1} d t=\int_{0}^{1} \sum_{\nu} x_{\nu} S^{r} d t=\sum_{\nu} \int_{0}^{1} x_{\nu} S^{r} d t
$$

Soit $S_{\nu}=S-x_{\nu}$. Alors

$$
\begin{gathered}
\int_{0}^{1} S^{r+1} d t=\sum_{\nu} \sum_{i=0}^{r}\left(\begin{array}{l}
r \\
i
\end{array}\right) \int_{0}^{1} x_{\nu} S_{\nu}^{i} x^{r-i} d t=\sum_{\nu} \sum_{i=0}^{r}\left(\begin{array}{l}
r \\
i
\end{array}\right) \int_{0}^{1} x_{\nu}^{r-i+1} d t \int_{0}^{1} S_{\nu}^{i} d t= \\
=\sigma_{r+1}+\sum_{\nu} \sum_{1}^{r}\left(\begin{array}{l}
r \\
i
\end{array}\right) \int_{0}^{1} x_{\nu}^{r-i+1} d t F_{i, \nu}+\sum_{\nu} \sum_{1}^{r}\left(\begin{array}{l}
r \\
i
\end{array}\right) \Theta_{i} H_{i} \int_{0}^{1} x_{\nu}^{r-i+1}
\end{gathered}
$$

où

$$
F_{i, \nu}=F_{i}\left(\sigma_{1}-\lambda_{\nu}^{(1)}, \ldots, \sigma_{i}-\lambda_{\nu}^{(i)}\right), \quad \lambda_{\nu}^{(s)}=\int_{0}^{1} x_{\nu}^{s} d t .
$$

Il en résulte que

$$
F_{i, \nu}=F_{i}\left(\sigma_{1}, \ldots, \sigma_{i}\right)-\lambda_{\nu}^{(1)} \frac{\partial F_{i}}{\partial \sigma_{1}}-\ldots-\lambda_{v}^{(i)} \frac{\partial F_{i}}{\partial \sigma_{i}}
$$

où

$$
\frac{\partial F_{i}}{\partial \sigma_{s}}=\frac{\partial F_{i}}{\partial \sigma_{s}}\left(\sigma_{1}, \ldots, \sigma_{s-1}, \sigma_{s}-\Theta \lambda_{v}^{(s)}, \sigma_{s+1}, \ldots, \sigma_{i}\right)
$$

Les arguments $\sigma_{1}^{*}, \sigma_{2}^{*}, \ldots$ étant bornés par $M$, on en conclut que $|\partial F| \partial \sigma_{s} \mid \leqslant K, \quad F_{i, \nu}=F_{i}\left(\sigma_{1}, \ldots, \sigma_{i}\right)+(r+1) \Theta \delta K$ et $\delta=\max \delta_{i}$, ce qui donne

$$
\begin{gathered}
\int_{0}^{1} S^{r+1} d t= \\
=\sigma_{r+1}+\sum_{\nu} \sum_{1}^{r}\left(\begin{array}{l}
v \\
i
\end{array}\right) \int_{0}^{1} x_{r}^{r-i+1} d t F_{i}\left(\sigma_{1}, \ldots, \sigma_{i}\right)+\delta(r+1) \Theta k+\Theta \sum_{1}^{r}\left(\begin{array}{l}
r \\
i
\end{array}\right) \sigma_{r-i+1}^{*} H_{i}= \\
=\sigma_{r+1}+\sum_{1}^{r}\left(\begin{array}{l}
r \\
i
\end{array}\right) \sigma_{r-i+1} F_{i}\left(\sigma_{1}, \ldots, \sigma_{i}\right)+\Theta H_{r+1} .
\end{gathered}
$$

On voit que

$$
F_{r+1}\left(\sigma_{1}, \ldots, \sigma_{r+1}\right)=\sigma_{r+1}+\sum_{i=1}^{r}\left(\begin{array}{l}
r \\
i
\end{array}\right) \sigma_{r-i+1} F_{i}\left(\sigma_{1}, \ldots, \sigma_{i}\right) .
$$


D'après le th. 3 de la I partie de ce travail ${ }^{\%}$ ), on peut trouver des constantes $K_{1}, K_{2}, \ldots$ telles qu'en désignant par $\lambda_{n, v}$ les médianes des fonctions $x_{n, v}$ et en posant

$$
x_{n, \nu}^{*}=\left\{\begin{array}{lll}
x_{n, \nu} & \text { si } & \left|x_{n, \nu}-\lambda_{n, \nu}\right| \leqslant K_{n} \\
0 & \text { si } & \left|x_{n, \nu}-\lambda_{n, \nu}\right|>K_{n}
\end{array}\right.
$$

on ait

$$
\int_{0}^{1}\left(\sum_{\nu} x_{n, \nu}^{*}\right)^{s} d t \rightarrow \int_{-\infty}^{\infty} x^{s} d V(x)=M_{s} \quad s=1,2, \ldots
$$

et, en particulier ${ }^{12}$ ),

$$
\sum_{\nu} \int_{0}^{1}\left(x_{n, v}^{*}-\Delta_{n, \nu}\right)^{2 s} d t \leqslant H_{s}, \quad \Delta_{n, \nu}=\int_{0}^{1} x_{n, \nu}^{*} d t .
$$

Il en résulte que

$$
\sum_{s}|\underset{t}{\mathrm{E}}| \infty_{n, \nu}^{*}-\Delta_{n, \nu}|>K|=O\left(K^{\cdots, s}\right) \quad . \quad s=1,2, \ldots
$$

Les inégalités (7.14) et (7.1) donnent $\max \left|\Delta_{n, v}\right| \rightarrow 0$, d'où l'on conclut en tenant compte de (7.14) que

$$
\sum_{\nu}\left|\underset{t}{\mathrm{E}}\left(\left|\infty_{n, v}^{*}\right|>K\right)\right|=O\left(K^{-8}\right) .
$$

Posons:

$$
\xi_{n, v}=\left\{\begin{array}{lll}
x_{n, \nu} & \text { si } & \left|x_{n, v}\right| \leqslant K_{n} / 2 \\
0 & \text { si } & \left|x_{n, v}\right|>K_{n} / 2 .
\end{array}\right.
$$

$D^{\prime}$ 'après le th. 3 de la $I$ partie $\left.{ }^{\theta}\right)$, il vient

$$
\int_{0}^{1}\left|\sum_{\nu} \xi_{n, \nu}\right|^{s} d t=O(1)
$$

et par conséquent:

$$
\begin{array}{ll}
\int_{0}^{1}\left(\sum_{v} \xi_{n, v}\right)^{s} d t \rightarrow M_{s}, & \int_{0}^{1}\left(\sum_{v} \xi_{n, v}-m\right)^{s} d t \rightarrow \dot{m}_{s}, \\
m=\int_{-\infty}^{+\infty} x d V(x), & m_{s}=\int_{-\infty}^{+\infty} x d V(x+m),
\end{array}
$$

12) J. Marcinkiewicz et A. Zygmund [10], p. 87-89, et [11], p. $109-113$. c. à d.

$$
\int_{0}^{1}\left\{\sum_{p}\left(\xi_{n, v}-m_{n, v}\right)\right\}^{s} d t \rightarrow m_{s}, \quad m_{n, v}=\int_{0}^{1} \xi_{n, v} d t
$$

ce qui donne

$$
\int_{0}^{1}\left(\sum_{\nu}\left(\xi_{n, \nu}-m_{n, \nu}\right)^{2}\right)^{s_{2}} d t=O(1)
$$

Il en résulte ${ }^{13}$ ) que

$$
\sum_{v} \int_{0}^{1}\left|\xi_{n, \nu}-m_{n, v}\right|^{s} d t=O(1)
$$

et, en particulier, que

$$
\int_{0}^{1}\left|\xi_{n, \nu}-m_{n, \nu}\right|^{s} d t=O(1)
$$

Or, comme $\max m_{n, v} \rightarrow 0$, il vient d'après (7.1)

$$
\max _{\nu} \int_{0}^{1}\left|\xi_{n, \nu}-m_{n, \nu}\right|^{s} d t \rightarrow 0 .
$$

En appliquant le lemme 9, on obtient

$\int_{0}^{1}\left[\sum_{\nu}\left(\xi_{n, \nu}-m_{n, \nu}\right]^{s} d t=F_{s}\left(\sigma_{1}^{(n)}, \sigma_{2}^{(n)}, \ldots, \sigma_{s}^{(n)}\right)+o(1) ; \quad \sigma_{s}^{(n)}=\sum_{\nu} \int_{0}^{1}\left(\xi_{n, v}-m_{n, \nu}\right)^{s}\right.$.

La dernière formule montre que le théorème est vrai lorsque les nombres $\sigma_{1}, \sigma_{2}, \ldots$ sont définis par les formules (7.11) et $m_{s}=F_{s}\left(\sigma_{1}, \ldots, \sigma_{s}\right)$. D'autre part, en appliquant les formules (7.5) et (7.6) à $e^{m z} \varphi(-i z)$, on démontre l'équivalence de (7.11) et (7.4), ce qui achève la démonstration:

\section{Errata.}

L'énoncé du th. 3 de la I partie de cet ourrage (Fundam. Math. 30, p. 209) contient une faute dans la définition des fonctions $x_{n, v}^{*}$ par la formule

$$
x_{n, \nu}^{*}=\left\{\begin{array}{lll}
x_{n, \nu} & \text { si } & \left|x_{n, v}\right| \leqslant K_{n} \\
0 & \text { si } & \left|x_{n, v}\right|>K_{n}
\end{array}\right.
$$

Cette formule est $\grave{a}$ corriger comme il suit:

$$
x_{n, \nu}^{*}=\left\{\begin{array}{lll}
x_{n, v} & \text { si } & \left|x_{n, \nu}-\lambda_{n, v}\right| \leqslant K_{n} \\
\lambda_{n, v} & \text { si } & \left|x_{n, \nu}-\lambda_{n, \nu}\right|>K_{n}
\end{array}\right.
$$

car autrement les fonctions $x_{n, y}^{\prime}-\lambda_{n, y}$ (p. 212) pourraient ne pas être bornées par $K_{n}$, ce qui rendrait impossible l'application du th. 1 de la p.203. On pent montrer même par des exemples très simples que le th. 3 , formulé comme il l'était, n'est pas vrai.

Remarquons toutefois que la définition primitive des fonctions $x_{n, v}^{*}$ peut être maintenue, lorsque les médianes de toutes les fonctions $x_{n, v}$ sont bornées par une constante $M$. Dans ce cas, les fonctions $x_{n, \nu}^{\prime}-\lambda_{n, v}$ (p. 212) sont bornées par $K_{n}+M$ et la démonstration reste vraie.

13) J. Marcinkiewiez et A. Zygmund [10] et [11], l. cit. 


\section{Travaux cités.}

[1] G. M. Bawly, Über einige Verallgemeinerungen der Grenzwertsätze der Wahrscheinlichkeitsrechnung, Recueil Math. 1 (1938), p. 917-930.

[2] A. Cournot, Théorie des chances et des probabilités, Paris 1843.

[3] H. Cramér, Random variables and probability distributions, Cambridge Tracts in Math. 36 (1937).

[4] B. Gnedenko, Über die Konvergenz der V'erteilungsgesetze von Summen voneinander unabhängiger Summanden, Comptes-Rendus Ac. Sc. U. R.S.S. 18 (1938), p. 231-234.

5] A. Khintchine, Zur Theorie der unbeschränlkt teilbaren Verteilungs. gesetze, Recueil Math. 2 (44) (1937), p. 80-119.

[6] P. Lévy, Calcul des probabilités, Paris 1925.

[7] P. Lévy, Variables aléatoires, Paris 1937.

[8] J. Marcinkiewicz, Sur les fonctions indépendantes I, Fund. Math. 30 (1938), p. 202-214.

[9] - Sur les fonctions indépendantes II, ibid., p. 347-364.

[10] J. Marcinkiewicz et A. Zygmund, Sur les fonctions indépendantes, Fund. Math. 29 (1937), p. 60-90.

[11] - Quelques théorèmes sur les fonctions indépendantes, Stud. Math. 7 (1937), p. 104-120

[12] G. Polya, Über den zentralen Grenzwertsatz der Wahrscheinlichkeits. rechnung und des Momentenproblem, Math. Zeitschr. 8 (1920), p. 157-181.

\section{Der Aussagenkalkül und die Topologie.}

Von

\author{
Alfred Tarski (Warszawa).
}

In dieser Arbeit möchte ich auf gewisse formale Verknüpfungen zwischen dem Aussagenkalkül und der Topologie (sowie einigen anderen mathematischen Theorien) hinweisen. Es handelt sich in erster Linie um eine topologische Deutung zweier Systeme des Aussagenkalküls, nämlich des üblichen (zweiwertigen) und des intuitionistischen (Brouwer-Heytingschen) Systems: jeder Aussage $\mathfrak{U}$ des Aussagenkalküls wird eine Aussage $\mathfrak{U}_{1}$ der Topologie in der Weise eineindeutig zugeordnet, daß $\mathfrak{U}$ dann und nur dann im zweiwertigen Kalkül beweisbar ist, wenn $\mathfrak{A}_{I}$ in jedem topologischen Raum gilt; eine analoge Übersetzungsvorschrift wird auch für den intuitionistischen Kalkül gegeben. Es scheint mir, daß die vorliegenden Betrachtungen nicht nưr vom rein formalen Gesichtspunkt aus ein gewisses Interesse bieten, sondern auch die inhaltliche Beziehung $z$ wischen den beiden Systemen des Aussagenkalküls and die ihnen zugrundeliegenden Intuitionen in interessanter Weise beleuchten.

Um etwaige Mißverständnisse zu vermeiden, möchte ich ausdrücklich bemerken, daß ich mich nicht bemüht habe, die verwendeten Schlußweisen den Forderungen der intuitionistischeṇ Logik anzupassen. $\left.{ }^{1}\right)^{2}$ )

\$1. Der zweiwertige und der intuitionistisehe Aussagenkalkül. Als Aussagenkalkül wird bekanntlich der elementarste Teil der mathematischen Logik bezeichnet. In den Ausdrücken des Aussagenkalküls kommt nur eine Art von Variablen vor, nämlich die sog. Aussagenvariablen, die ganze Aussagen 